\title{
Impact of the transient formation of molecular hydrogen on the microcrack nucleation and evolution in H-implanted Si (001)
}

S. Personnic, K. K. Bourdelle, F. Letertre, A. Tauzin, N. Cherkashin, A. Claverie, R. Fortunier, and H. Klocker

Citation: Journal of Applied Physics 103, 023508 (2008); doi: 10.1063/1.2829807

View online: https://doi.org/10.1063/1.2829807

View Table of Contents: http://aip.scitation.org/toc/jap/103/2

Published by the American Institute of Physics

\section{Articles you may be interested in}

Development of microcracks in hydrogen-implanted silicon substrates

Journal of Applied Physics 114, 123513 (2013); 10.1063/1.4821239

Investigation of the cut location in hydrogen implantation induced silicon surface layer exfoliation Journal of Applied Physics 89, 5980 (2001); 10.1063/1.1353561

Effect of the order of $\mathrm{He}^{+}$and $\mathrm{H}^{+}$ion co-implantation on damage generation and thermal evolution of complexes, platelets, and blisters in silicon

Journal of Applied Physics 119, 135308 (2016); 10.1063/1.4945032

Study of the formation, evolution, and dissolution of interfacial defects in silicon wafer bonding Journal of Applied Physics 107, 093513 (2010); 10.1063/1.3357389

Study of extended-defect formation in $\mathrm{Ge}$ and $\mathrm{Si}$ after $\mathrm{H}$ ion implantation

Applied Physics Letters 86, 181910 (2005); 10.1063/1.1906319

Cracks and blisters formed close to a silicon wafer surface by He-H co-implantation at low energy Journal of Applied Physics 118, 245301 (2015); 10.1063/1.4938108

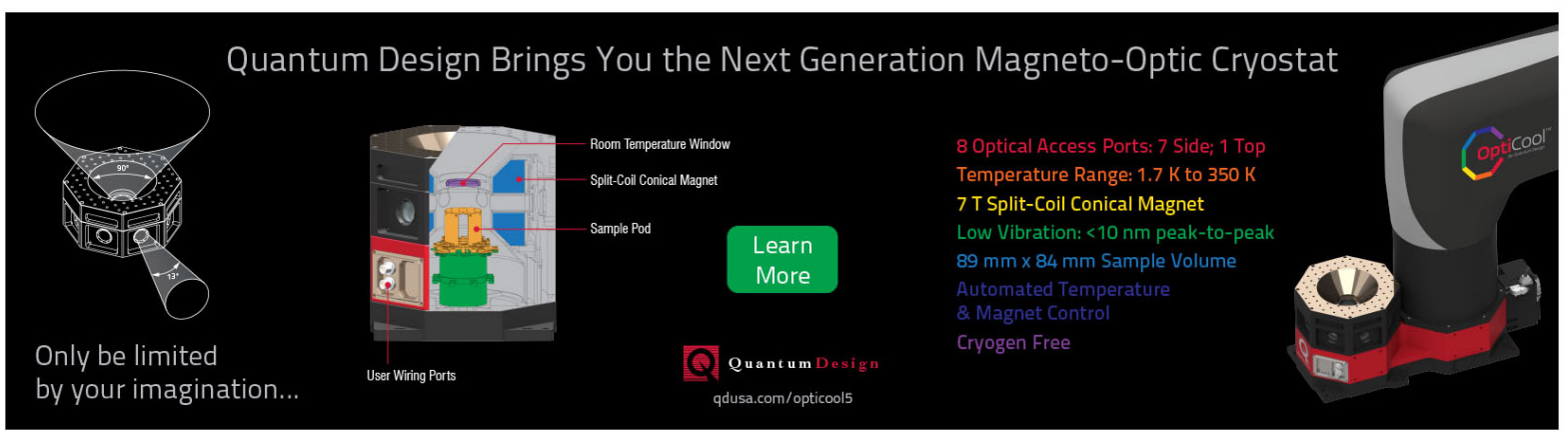




\title{
Impact of the transient formation of molecular hydrogen on the microcrack nucleation and evolution in H-implanted Si (001)
}

\author{
S. Personnic, K. K. Bourdelle, ${ }^{a)}$ and F. Letertre \\ Silicon-on-Insulator Technologies (SOITEC), Parc Technologique des Fontaines, 38926 Crolles Cedex, \\ France
}

\author{
A. Tauzin \\ CEA-DRT-LETI, CEA-GRE, 17 Rue des Martyrs, 38054 Grenoble Cedex 9, France
}

N. Cherkashin and A. Claverie

CEMES/CNRS, nMat Group, BP 4347, F-31055 Toulouse, France

R. Fortunier and $\mathrm{H}$. Klocker

EMSE, 158 Cours Fauriel, 42023 Saint-Etienne Cedex 2, France

(Received 17 September 2007; accepted 7 November 2007; published online 22 January 2008)

\begin{abstract}
We study the implant-induced hydrogenated defects responsible for the Smart Cut ${ }^{\mathrm{TM}}$ layer transfer of Si (001) films. Different experimental methods are used to quantify the time dependence of the defect evolution and interactions during isothermal annealings. An optical characterization technique was developed for the statistical analysis of the formation and growth of micrometer size microcracks in the buried implanted layer. We show that the formation of molecular hydrogen is dominated by a transient phenomenon related to the rapid dissociation of the hydrogenated point defects. The impact of the $\mathrm{H}_{2}$ formation kinetics on the microcrack evolution is described and the physical mechanisms involved in their growth are identified. A comprehensive picture of the fracture phenomenon in $\mathrm{H}$ implanted Si leading to the full layer transfer is proposed and discussed. (C) 2008 American Institute of Physics. [DOI: 10.1063/1.2829807]
\end{abstract}

\section{INTRODUCTION}

The discovery of the way to fabricate silicon-oninsulator (SOI) structures with the Smart Cut ${ }^{\mathrm{TM}}$ technology ${ }^{1}$ has significantly enhanced the scope of applications of ion implantation. Hydrogen among other species is used to produce SOI wafers. It is well known that $\mathrm{H}$ is mobile and has a strong chemical activity in crystalline $\mathrm{Si}^{2}{ }^{2}$ During $\mathrm{H}$ implantation a large variety of defects are created. For the room temperature implants the hydrogenated point defects (HPDs) are represented largely by the vacancy-hydrogen complexes. In addition, two-dimensional defects named platelets are formed in the implant-induced damage zone. ${ }^{3}$ It has been shown by transmission electron microscopy (TEM) that during thermal annealing these defects undergo an Oswald ripening process interchanging the $\mathrm{H}$ atoms they contain. ${ }^{4}$ The corresponding growth of the platelets induces the formation of large microcracks in the narrow depth region around project range of the $\mathrm{H}$ ions, $R_{p}{ }^{5}$ The propagation and interaction of the microcracks lead to the full layer transfer of $\mathrm{Si}$ in the presence of a stiffener. ${ }^{1,5}$ Despite significant efforts, many physical aspects of the evolution of the hydrogenated defects from clusters to platelets then to microcracks are still unclear.

In this article, we present a time-dependence study of the hydrogenated defects responsible for the transfer of $\mathrm{Si}(001)$ thin layers during isothermal treatments. In the text we will distinguish the following.

HPDs, i.e., the multivacancies $V_{m} \mathrm{H}_{n}$, the monovacan-

${ }^{a)}$ Electronic mail: konstantin.bourdelle@ soitec.fr. cies $V \mathrm{H}_{n}$, and the $\mathrm{H}_{2}{ }^{*}$ defects $\left(\mathrm{Si}-\mathrm{H}_{\mathrm{AB}}\right.$ and $\left.\mathrm{Si}-\mathrm{H}_{\mathrm{BC}}\right)$. (ii) Hydrogenated extended defects, HEDs, i.e., platelets and microcracks.

Different experimental methods are used to quantify defect evolution and interactions. An optical characterization technique has been developed for the statistical analysis of the buried microcracks. We show that the formation of hydrogen molecules during annealing is dominated by a transient phenomenon related to the rapid dissociation of the specific HPDs. The impact of the $\mathrm{H}_{2}$ formation kinetics on the HEDs evolution is investigated and the physical mechanisms involved in their growth are identified. The presented results elucidate and quantify many aspects of the fracture phenomenon in $\mathrm{H}$ implanted Si (001).

\section{EXPERIMENTS}

$200 \mathrm{~mm}$ p-type czochralski silicon (001) substrates were implanted with $\mathrm{H}$ at an energy of about $40 \mathrm{keV}$ and a dose of a few $10^{16} \mathrm{~cm}^{-2}$ through a $145 \mathrm{~nm}$ thick thermally grown oxide. After implantation and hydrophilic bonding to a base substrate, the structures were cut into sample pieces. These samples underwent annealing at temperatures between 350 and $500{ }^{\circ} \mathrm{C}$ for different times $t_{a}$ ranging from $30 \mathrm{~s}$ to $51 \mathrm{~h}$.

In a previous study ${ }^{6}$ we measured the time needed to obtain the full layer transfer at a given temperature: the splitting time $t_{s}(T)$. The data showed an Arrhenius-type dependence with an activation energy $E_{a}=2.3 \pm 0.1 \mathrm{eV}$. In this paper, in order to compare results obtained at different temperatures, the annealing time will be also expressed as fraction or percentage of $t_{s}(T)$. 
Different physical characterization techniques were used to track the behavior of implanted hydrogen and the evolution of the HPDs and HEDs during annealing. The $\mathrm{H}$ depth profiles were obtained by secondary ion mass spectroscopy (SIMS) technique using the $\mathrm{MCs}_{2}{ }^{+}$method suitable for analysis of light electronegative elements. ${ }^{7}$ A Cameca IMS-5F apparatus with a $2 \mathrm{keV} \mathrm{Cs}^{+}$sputtering beam operated under a vacuum of $\sim 1 \times 10^{-9}$ Torr in the sample chamber. The elastic recoil detection analysis (ERDA) with a 2.3 MeV He analyzing beam was performed to monitor the total amount of $\mathrm{H}$ retained in the samples.

Fourier transform infrared spectroscopy (FTIR) was carried out in the multiple internal reflection mode with a Brucker IFS 55 spectrometer (resolution of $4 \mathrm{~cm}^{-1}$ ) to quantify the types and relative distributions of hydrogenated defects in the as-implanted and annealed samples. Fitting of the FTIR spectra was performed using the GRAMS program. ${ }^{8}$ To deconvolute the spectra, we considered the well-known stretching modes for $\mathrm{Si}-\mathrm{H}_{n}$ defects. ${ }^{2,9-11}$ Following the analysis in Ref. 2 we assumed that the absorption cross sections for the infrared light are the same for the different $\mathrm{Si}-\mathrm{H}_{n}$ chemical bonds in the range of $1800-2250 \mathrm{~cm}^{-1}$. The intensity of a given stretching mode is then directly proportional to the amount of hydrogen in the corresponding configuration. The peaks in the spectrum were fitted by a Gaussian-type profile with the width corresponding to the resolution of the spectrometer. For the quantitative analysis we have combined the peaks into several bands. That eliminates some uncertainties related to the assignment of certain $\mathrm{Si}-\mathrm{H}_{n}$ modes.

The platelet populations were analyzed by cross sectional transmission electron microscopy (XTEM) mostly under out-of-Bragg strongly out-of-focus conditions, suitable for the Fresnel imaging of these phase objects. A JEOL 2010 electron microscope operating at $200 \mathrm{keV}$ was used. The XTEM characterization was restricted to the samples annealed for the times shorter than $50 \%$ of $t_{s}(T)$. For longer annealings, the samples become very fragile and cannot be prepared using standard techniques. With more complicated preparation procedures involving the encapsulation of the overall structure and sample thinning by focused ion beam, large microcracks, up to $1-2 \mu \mathrm{m}$ in diameter, have also been observed. ${ }^{5}$ The statistical analysis of these defects by XTEM is, however, not possible.

To image the buried microcracks we used a Wyko NT 3300 optical profiler in a phase shifting interferometry mode with a white-light beam filtered at $632 \mathrm{~nm}$. The sample preparation for this analysis was as follows. After $\mathrm{H}$ implantation, a $\mathrm{SiO}_{2}$ film was deposited onto the wafers using the plasma enhanced chemical vapor deposition technique. The deposition temperature was kept below $200{ }^{\circ} \mathrm{C}$ in order to avoid $\mathrm{H}$ redistribution. For deposited thickness larger than $10 \mu \mathrm{m}$ the $\mathrm{SiO}_{2}$ stiffener completely prevented formation of blisters at the Si surface during annealing, leading to a full layer transfer. The corresponding $t_{s}(T)$ was exactly the same as for the bonded SOI structure with a Si wafer as a stiffener. Atomic force microscopy showed no difference in postsplit surface roughness between bonded SOI and the structure with deposited $\mathrm{SiO}_{2}$ layer as a stiffener. Due to transparency

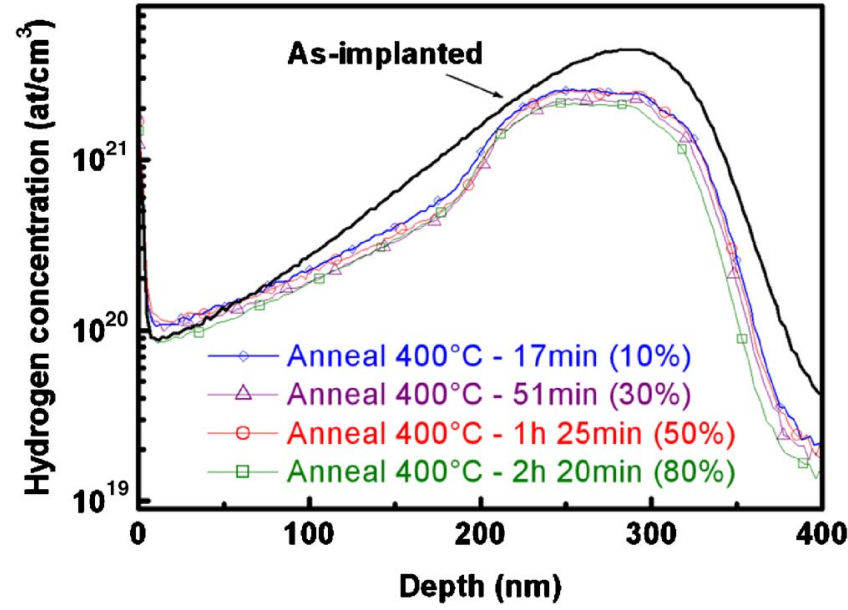

FIG. 1. (Color online) Hydrogen SIMS profiles before and after isothermal annealings at $400{ }^{\circ} \mathrm{C}$.

of the $\mathrm{SiO}_{2}$ film at the wavelength of $632 \mathrm{~nm}$ and relatively small absorption in Si the observation of buried microcracks is possible. The beam of the profiler can be focused either at the depth region where the microcracks develop or at the surface of the $\mathrm{SiO}_{2}$ stiffener. The microcracks with a size larger than $1 \mu \mathrm{m}$ can be imaged using this technique. Laser marking of the sample surface allows observation of the evolution of the same population of the microcracks.

Some companion samples were annealed without any oxide layer on the Si surface to induce the formation of blisters. For such samples, the optical profiler was used to measure the diameter and height of the blisters. The corresponding volume and amount of the molecular hydrogen contained in the blisters was estimated based on the approach developed in Refs. [12,13].

\section{RESULTS AND INTERPRETATION}

\section{A. Time dependence of molecular $\mathrm{H}_{2}$ formation}

Figure 1 shows the evolution of the $\mathrm{H}$ depth distribution during annealing. Two main features are observed. First, there is a "transient" signature: changes in the depth profile occur only during initial stage of the anneal, i.e., for $t_{a}$ $<t_{s}(T) / 10$. The redistribution shows the evidence of an "uphill diffusion:" the concentration gradients at the slopes of the profile increase after annealing while a plateau is observed in the $R_{p}$ region. These are classical features observed when the precipitation of a second phase from a supersaturated matrix occurs since the precipitates tend to absorb the free mobile species around them. Second, in agreement with our previous report, ${ }^{6}$ we note an apparent $\mathrm{H}$ "dose loss": reduction in the integrated area under the profile after annealing. That is not consistent with the small redistribution of $\mathrm{H}$ in the surface region and might be due to $\mathrm{H}$ precipitation in a form that is not or only partially detected by SIMS.

To verify this assumption we have undertaken additional experiments with the ERDA technique sensitive to the total hydrogen content in the sample regardless of the chemical state. The normalized amounts of $\mathrm{H}$ determined by SIMS and ERDA are plotted in Fig. 2 as a function of percentage of $t_{s}(T)$ for two temperatures. It is seen that the amount of hy- 


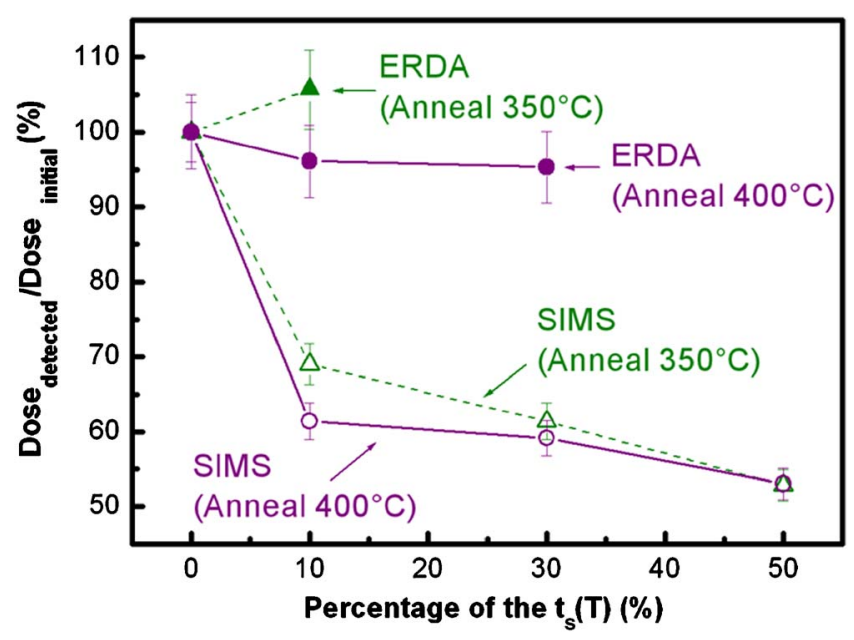

FIG. 2. (Color online) Normalized hydrogen dose measured by SIMS and ERDA as a function of percentage of $t_{s}(T)$ for isothermal annealings at 350 and $400{ }^{\circ} \mathrm{C}$.

drogen detected by SIMS strongly decreases during the first stage of annealing, whereas the ERDA signal does not change significantly with time. This is a clear confirmation that the decrease of the SIMS signal seen in Fig. 1 is not due to the outdiffusion of $\mathrm{H}$ from the implanted region but to some rearrangement of the $\mathrm{H}$ atoms in a form not detectable by SIMS.

Figure 3 shows that the fast rearrangement of the hydrogenated defects measured by FTIR also occurs during the initial stage of annealing. The variation of the integrated intensity of the FTIR signal along with the normalized dose obtained by SIMS is depicted in Fig. 4 as a function of the percentage of $t_{s}(T)$. A similar transient behavior is observed, although the amplitude drop is more pronounced for the FTIR signal.

Using the deconvolution procedure described in Sec. II the time evolution of the different types of hydrogenated defects was extracted. The normalized intensity values (annealed at $450{ }^{\circ} \mathrm{C}$ ) are shown in Fig. 5 for: (a) the multivacancy defects $V_{m} \mathrm{H}_{n}$ (broadband between 1880 and $2025 \mathrm{~cm}^{-1}$ ); (b) the monovacancy defects $V \mathrm{H}_{3,4}$ (band be-

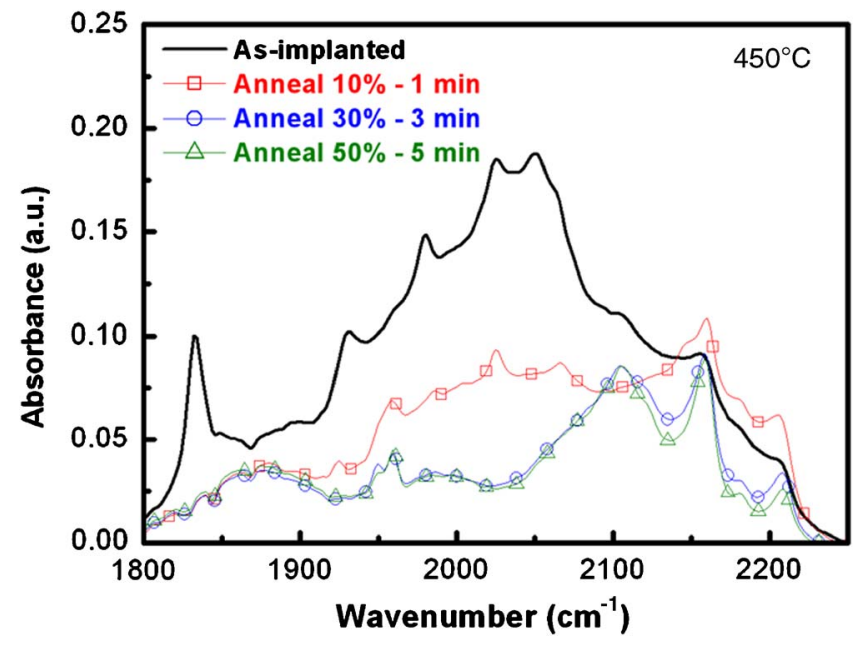

FIG. 3. (Color online) FTIR spectra of the $\mathrm{Si}-\mathrm{H}$ stretching modes in $\mathrm{H}$ implanted $\mathrm{Si}$ before and after isothermal annealings at $450{ }^{\circ} \mathrm{C}$.

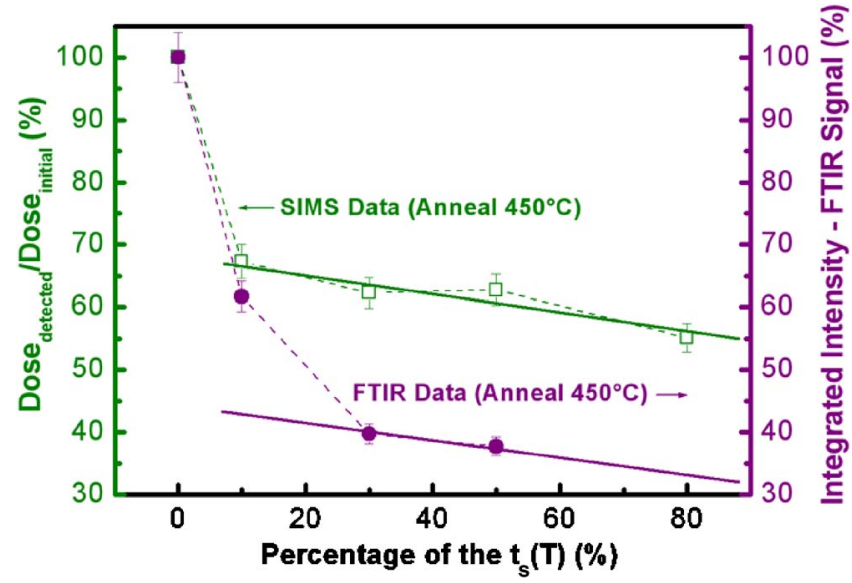

FIG. 4. (Color online) Normalized SIMS dose and integrated intensity of FTIR spectra as a function of percentage of $t_{s}(T)$ for isothermal annealings at $450{ }^{\circ} \mathrm{C}$.

tween 2160 and $2210 \mathrm{~cm}^{-1}$ ); (c) the $\mathrm{H}_{2}^{*}$ defects (modes at 1832 and $2052 \mathrm{~cm}^{-1}$ ); (d) $V \mathrm{H}_{2}$ defects and the $\mathrm{H}$ passivating the internal (100) surfaces (modes between 2085 and $2130 \mathrm{~cm}^{-1}$ ). Two very different types of behavior are observed. On one hand [Figs. 5(a) and 5(c)], the concentrations of multivacancies and $\mathrm{H}_{2}^{*}$ defects rapidly decrease during the first $30 \%$ of $t_{s}(T)$. There is little change in concentration of these defects for longer annealing times. On the other hand, Fig. 5(b) indicates that only a small fraction of the initially predominant $V_{m} \mathrm{H}_{n}$ defects, $\sim 3 \%$ of the implanted H-dose, might be converted into $\mathrm{VH}_{3,4}$ defects during the initial stage of annealing. The details of the evolution of the intensity of the modes related to $V \mathrm{H}_{3,4}$ defects will be discussed in Sec. III B together with the XTEM data.

The decrease of the infrared band around $2100 \mathrm{~cm}^{-1}$ during transient stage may be associated with the dissociation of the $V \mathrm{H}_{2}$ defects. The corresponding stretching mode is in the same wave number range, i.e., around $2121 \mathrm{~cm}^{-1}$. ${ }^{9}$ Myers et $a l .{ }^{14}$ found that the binding energy of hydrogen with vacancies is smaller than that for hydrogen passivating the internal surfaces. Therefore the dissolution of $V \mathrm{H}_{2}$ defects is energetically favorable in agreement with the information obtained from the deconvolution of the infrared spectra [Fig. $5(\mathrm{~d})]$.

Theoretical calculations ${ }^{15}$ indicate that $\mathrm{H}_{2}$ can be stable at the tetrahedral interstitial $T_{d}$ sites. Other reports demonstrated that the existence of point defects in Si promotes the dissociation of $\mathrm{H}_{2},{ }^{16}$ with a dissociation rate increasing with the degree of the crystalline disorder. ${ }^{17}$ Our FTIR data show that during annealing the morphology of the implant damage changes, notably during the initial stage of the annealing, leading to an overall strong decrease of the HPD concentration. We cannot rule out that this transformation can produce molecular $\mathrm{H}_{2}$ stable at the $T_{d}$ sites. The solubility of $\mathrm{H}_{2}$ in $\mathrm{Si}$ at room temperature is, however, negligibly small. ${ }^{2}$ The concentration of $\mathrm{H}_{2}$ located on the $T_{d}$ sites must be, therefore, very low.

The combination of SIMS, ERDA, and FTIR data can be explained by the rapid conversion of hydrogen trapped within HPDs in the as-implanted state into molecular $\mathrm{H}_{2}{ }^{2}$ 

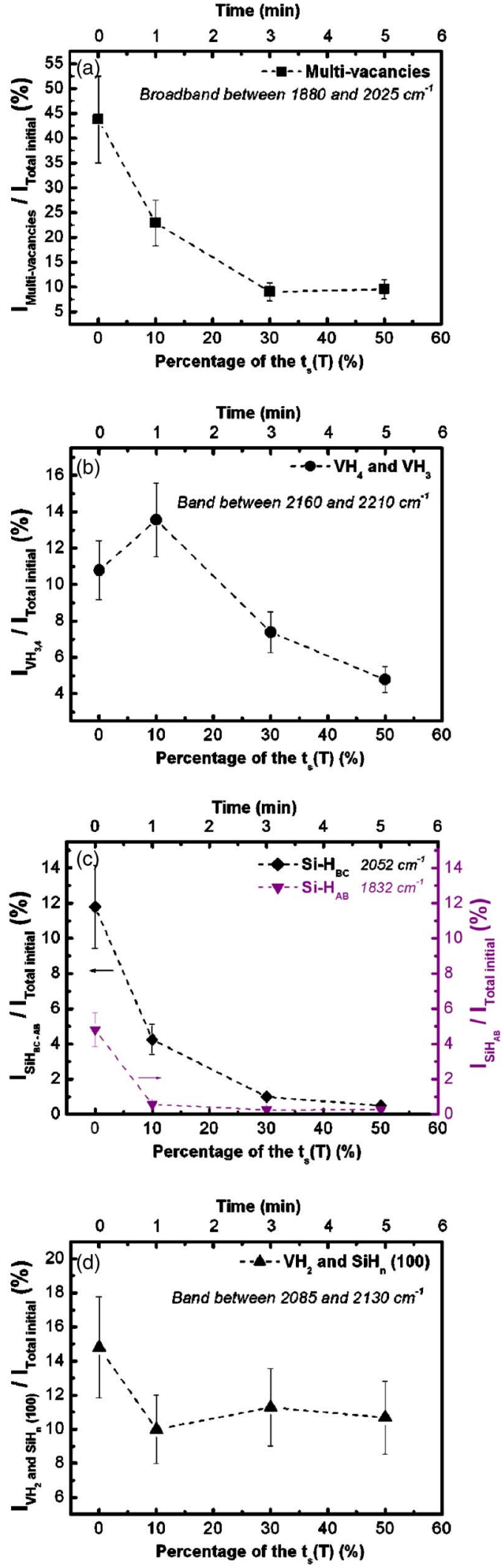

FIG. 5. (Color online) Evolution of the multivacancy (a), monovacancy (b), $\mathrm{H}_{2}^{*}$ (c), and $\mathrm{H}$ passivating (100) internal surfaces (d) FTIR modes during annealing at $450^{\circ} \mathrm{C}$.

We believe that the observed SIMS dose loss is due to the limited capability of this technique to detect molecular $\mathrm{H}_{2}$. The transient time domain during which the dissociation/ recombination of the multivacancies and $\mathrm{H}_{2}^{*}$ defects takes

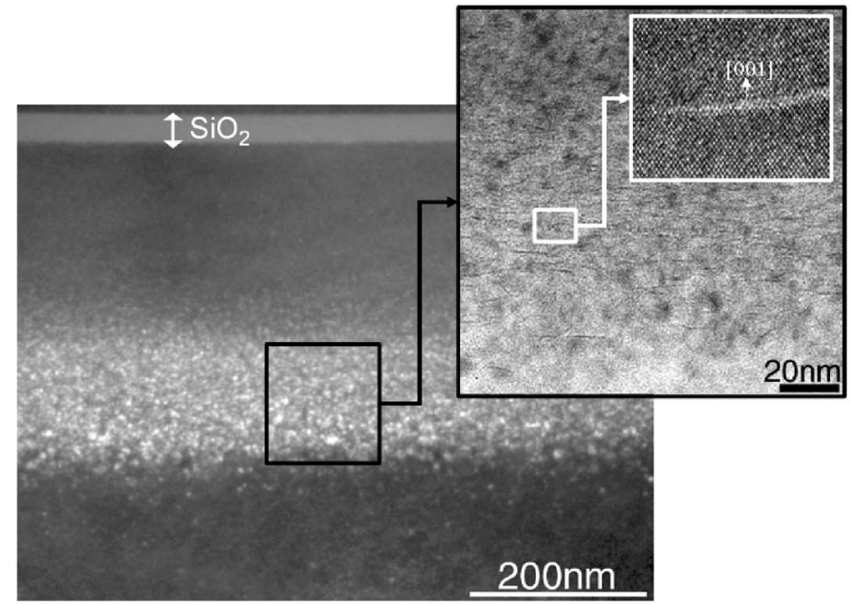

FIG. 6. TEM micrographs of the damage zone (a), platelets population (b), and (001) platelet parallel to the wafer surface (c).

place can be directly related to the precipitation of hydrogen in molecular form. In our opinion, the high pressure of $\mathrm{H}_{2}$ within the HEDs (Ref. 4) as well as the high ionization cross section for $\mathrm{H}_{2}$ limits the capability of the SIMS technique to detect hydrogen trapped in these defects. During advancement of the sputtering crater in the condition of high vacuum the pressurized gaseous $\mathrm{H}_{2}$ quickly leaves the area of ionization drastically decreasing the SIMS yield.

It is rather difficult to determine the amount of molecular hydrogen in the as-implanted state. With our data, however, it is possible to quantify the amount of molecular $\mathrm{H}_{2}$ after the end of the transient stage. From the reduction of the SIMS signal, and FTIR data we can deduce that the HEDs contain more than $70 \%$ of the molecular $\mathrm{H}_{2}$ created during the thermal treatment. A smaller fraction of $\mathrm{H}_{2}$ molecules, less than $30 \%$, corresponding to the difference between SIMS and FTIR signals might be trapped into small clusters such as the stable $V_{m} \mathrm{H}_{n}$ defects and, in minor proportion, is located in the $T_{d}$ sites of the $\mathrm{Si}$ matrix.

Analysis of the integrated intensity of the FTIR spectra shows that the amount of molecular hydrogen formed after long annealing, $80 \%$ of $t_{s}(T)$, corresponds to $\sim 60 \%-65 \%$ of the implanted dose. We assume here that $\mathrm{H}$ not detected by FTIR in the form of $\mathrm{SiH}_{n}$ defects is in molecular form since ERDA shows negligible outdiffusion of $\mathrm{H}$ from the implanted region. Figure 5(a) indicates that a fraction of $V_{m} \mathrm{H}_{n}$ defects, corresponding to about $10 \%$ of the implanted dose, remains stable upon this long time annealing. We believe that a number of $\mathrm{H}_{2}$ molecules might be trapped within the clusters created by the more stable $V_{m} \mathrm{H}_{n}$ defects. The existence of such configuration has been observed in the other reports. ${ }^{18-20}$

\section{B. Platelets nucleation and growth: XTEM analysis}

XTEM was used to quantitatively follow the evolution of the characteristics of the populations of platelets during annealing of the same set of samples. A typical damage zone was observed between 170 and $370 \mathrm{~nm}$ below Si surface and the fracture plane was at a depth of about $300 \mathrm{~nm}$ [Fig. 6(a)]. Two families of platelets were found with habit planes either 

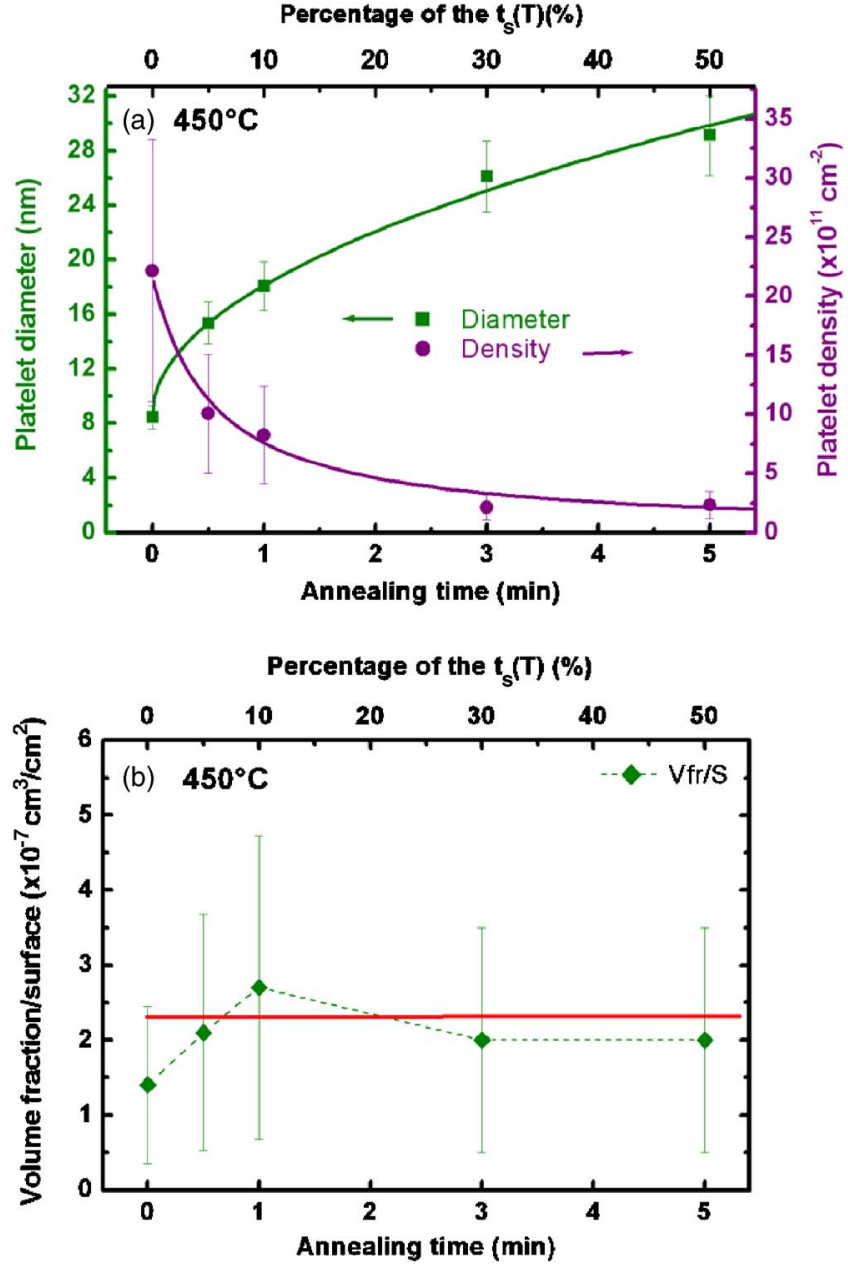

FIG. 7. (Color online) Evolution of mean diameter and surface density (a) and platelet volume fraction per unit area (b) as a function of the annealing time at $450{ }^{\circ} \mathrm{C}$.

lying on the (001) plane parallel to the wafer surface [Figs. $6(\mathrm{~b})$ and $6(\mathrm{c})]$ or on two sets of (111) plane. Under our experimental conditions, the (111) platelets are located deeper in the damage zone and are detected in considerably smaller densities than the (001) platelets. Using the method described in details in Ref. 4, we have carried out a statistical analysis of the characteristics of the platelet populations found after each annealing step. Figure 7(a) presents the time evolution of the mean size and the density of the (001) platelets during annealing at $450{ }^{\circ} \mathrm{C}$. From these results, the surface and the volume fractions the platelet population can be extracted [Fig. 7(b)].

It is striking to note that this surface fraction increases again during the first stage of the annealing. Since the density of platelets does not increase in the mean time, the nucleation stage of the platelets is already completed after implantation. During the transient stage, for $t_{a}<10 \%$ of $t_{s}(T)$, the platelets are involved in a "pure growth" regime during which the existing nuclei grow by "pumping" $\mathrm{H}$ from other less stable defects in the matrix, invisible by TEM. This is consistent with the FTIR and SIMS results showing a dramatic drop of the integrated signal intensities also during the same transient stage. Only the IR band corresponding to the monovacancies defects $\mathrm{VH}_{3,4}$ increases in amplitude dur- ing this period of time [Fig. 5(b)]. Since these $\mathrm{VH}_{3,4}$ defects are thought to be the precursors of the platelets, 2,21 our IR data show that only $3 \%$ of the implanted dose is used for formation of new platelets during the transient stage of annealing.

The combination of FTIR, SIMS, and TEM results leads to the following scenario for the platelets growth. The $\mathrm{H}_{2}$ molecules are produced mostly following the dissociation of HPDs and the migration of $\mathrm{H}$ toward the platelets during the transient period. As the platelets grow by the injection of $\mathrm{H}$ from other less stable defects, the pressure inside them quickly increases leading to their propagation. Later on, for $t_{a}>10 \%$ of $t_{s}(T)$, the average diameter of the platelets still increases and their density decreases in such a manner that the surface and volume fractions per unit area they occupy remain nearly constant. These characteristics are typical of a conservative Ostwald ripening mechanism ${ }^{4}$ during which the platelets interchange the species they are composed of, namely, hydrogen atoms and vacancies. This scenario is consistent with the FTIR observations where the intensity of the stretching modes around $2100 \mathrm{~cm}^{-1}$ assigned to $\mathrm{H}$ passivating the internal (100) surfaces of the platelets remains constant during the same period of time [Fig. 5(d)].

\section{Microcrack evolution}

The characterization of annealed samples by XTEM is restricted to times shorter than $50 \%$ of $t_{s}(T)$ for which the maximum platelet diameter is of about $40 \mathrm{~nm}$. The optical characterization of the microcrack evolution at $\sim 300 \mathrm{~nm}$ below the Si surface was therefore performed using phase shifting interferometry technique described in Sec. II.

Figure 8(a) shows a typical image of the buried microcracks obtained after annealing of the implanted wafers covered by a thick $\mathrm{SiO}_{2}$ layer, for $t_{a}$ corresponding to $40 \%$ of $t_{s}(T)$. Clearly, this technique evidences the presence of a population of microcracks with sizes ranging from a few to several micrometers and with a density of the order of $10^{6} \mathrm{~cm}^{-2}$. No blisters are observed for such conditions as confirmed by imaging of the wafer surface [Fig. 8(b)]. Figure 9 shows the isothermal evolution of the microcracks at $350{ }^{\circ} \mathrm{C}$. Two distinct phenomena are observed. First, the smallest microcracks dissolve (circles in Fig. 9), while the largest grow. This behavior suggests that microcracks are also involved in Ostwald ripening, i.e., interchange the vacancies and hydrogen atoms they are composed of. The growth of the largest microcracks, however, occurs also, in part, through the coalescence between neighboring, already large, microcracks (squares in Fig. 9). This phenomenon does not involve the motion of these defects but instead appears as the result of the sudden overlapping of their strain fields as they grow by the regular Ostwald ripening mechanism. Such a geometric coalescence leads to the random formation of large microcracks with the size up to tens of micrometers after annealing for $50 \%$ of $t_{s}(T)$.

This growth mechanism gives rise to the formation of microcracks of irregular shapes, in contrast with the circular shape of the platelets. We have performed a statistical analysis of such images, measuring the surface area occupied by 

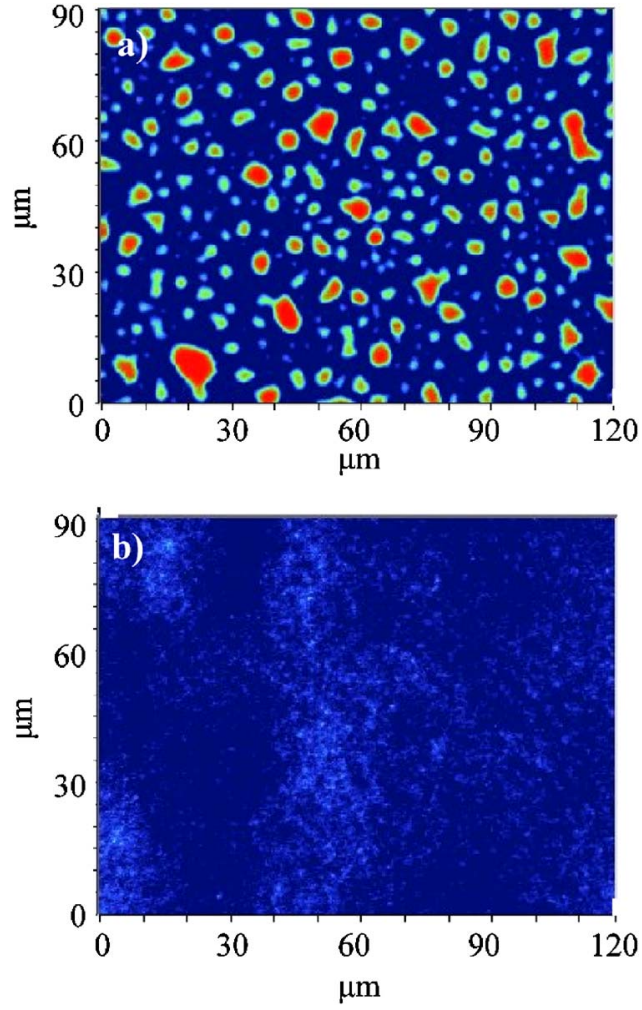

FIG. 8. (Color online) Optical profiler images obtained for the wafer with a thick stiffener after annealing at $400{ }^{\circ} \mathrm{C}$ : buried microcracks (a) and the surface of the deposited $\mathrm{SiO}_{2}$ layer (b).

the microcracks and assigning them the mean diameter they would have if circular in shape. Figure 10 depicts the size distributions of the microcracks for different isothermal annealings at $400{ }^{\circ} \mathrm{C}$. It is striking to note that, for $t_{a} \geqslant 50 \%$ of $t_{s}(T)$, the distribution becomes bimodal. The amplitude of the first peak decreases during annealing while the relative contribution of the second increases. The position of the first peak only slightly shifts toward larger diameters during the fist stage of annealing. That indicates that the contribution of the Ostwald ripening mechanism to the overall growth of the microcracks is marginal. Alternatively, the relative increase of the second peak, centered at the size slightly larger than twice that of the first peak, is the signature of the coalescence process.

The important conclusions from this analysis are that microcrack coalescence occurs during their "regular" growth by Ostwald ripening and that this coalescence process is almost conservative. These first findings are confirmed by the
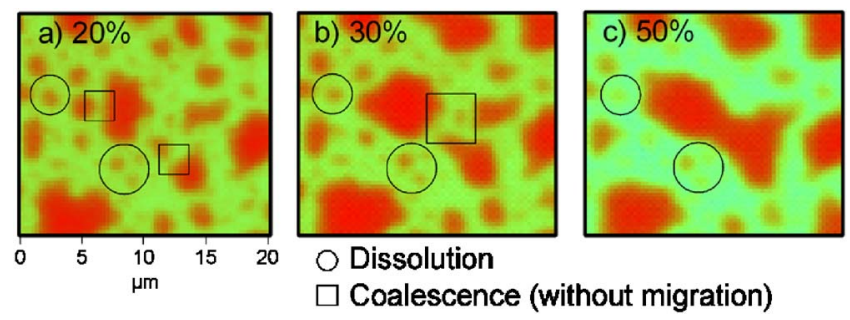

FIG. 9. (Color online) Optical profiler images of buried microcracks after an annealing at $350{ }^{\circ} \mathrm{C}$ for $20 \%$ (a), 30\% (b), and $50 \%$ (c) of $t_{s}(T)$. The images illustrate the Oswald ripening and the coalescence mechanisms.

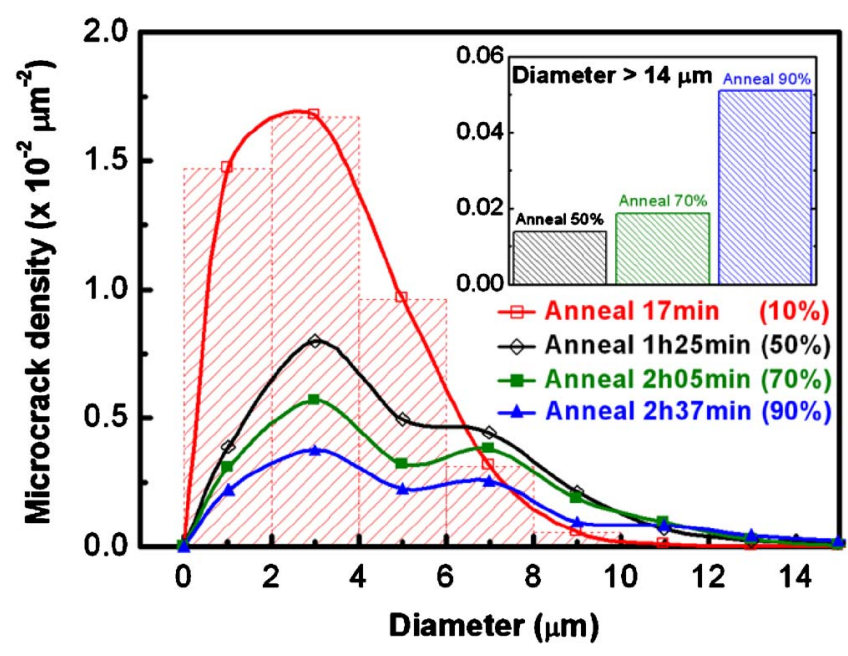

FIG. 10. (Color online) Size distributions of the microcracks after different annealings at $400{ }^{\circ} \mathrm{C}$.

quantitative analysis of the data shown in Fig. 11. For annealing times ranging from $10 \%$ to $80 \%-90 \%$ of $t_{s}(T)$, the mean diameter and the inverse of the density of microcrack population show a linear dependence on the square root of the time and on the time, respectively [Figs. 11(a) and 11(b)]. This time dependence is characteristic of the Ostwald ripening for two-dimensional precipitates. During the same time interval the surface fraction covered by these defects remains constant, at about $30 \%$ of the total surface of the wafer [Fig. 11(c)]. The increase seen at the beginning of the annealing, from $10 \%$ to $20 \%$ of $t_{s}(T)$, might be due to the growth of some microcracks which sizes were below the detection limit, i.e., $1 \mu \mathrm{m}$. We cannot rule out also the spontaneous formation (nucleation) of new microcracks.

\section{Driving force behind microcrack growth}

Using the SIMS technique we have shown previously ${ }^{6}$ that the kinetics of $\mathrm{H}_{2}$ formation in HEDs is close to that for the layer transfer. It has been suggested by Freund ${ }^{22}$ that the growth of an individual microcrack is driven by the pressure of the hydrogen gas. In order to verify whether the quantitative analysis presented in Secs. III B and III C is consistent with this assumption, we simulated the dependence of the individual microcrack radius on the annealing time at $400{ }^{\circ} \mathrm{C}$.

The microcracks growth by the Oswald ripening mechanism implies that the pressure of $\mathrm{H}_{2}$ within the larger ones increases due to the arrival of hydrogen from the smaller ones. When the magnitude of the gas pressure exceeds the critical value $P_{c}$ the microcrack propagation occurs. The increase of the microcrack diameter can be modeled based on Griffith's criterion. ${ }^{22}$ From a simple energy balance calculation, the $P_{c}$ value can be obtained as a function of the silicon properties and the microcrack radius.

Assuming a uniform pressure of $\mathrm{H}_{2}$ molecules within the defect, Griffith's criterion can be formulated as follows: 


$$
\frac{d}{d R}\left(-\frac{4 \pi(1-2 \nu) P^{2} R^{3}}{3 E}+2 \pi \gamma_{\mathrm{Si}} R^{2}\right)<0
$$

where $E$ is Young's modulus of silicon, $\gamma_{\mathrm{Si}}$ is the surface energy density, $\nu$ is the Poisson ratio, $R$ is the radius of the microcrack, and $P$ is the pressure of $\mathrm{H}_{2}$ molecules. This equation gives the conditions for the microcrack propagation. The first part corresponds to the mechanical energy of a microcrack, and the second to the energy of the additional fracture surface.

The pressure can be estimated using the ideal gas law since the volume formed by the $\mathrm{H}_{2}$ molecules is significantly smaller than that for the microcrack,

$$
P=\frac{N_{\mathrm{H} 2} k T}{\pi R^{2} h},
$$

where $h$ is the microcrack thickness, $k$ is Boltzman's constant, and $N_{\mathrm{H} 2}$ the number of hydrogen molecules in the microcrack.

To use Griffith's criterion we need to estimate the number of $\mathrm{H}_{2}$ molecules within an individual microcrack. The amount of molecular hydrogen within the HEDs in the damage zone can be obtained, e.g., with the SIMS technique. To estimate the amount of $\mathrm{H}_{2}$ contained in the microcracks located in the immediate vicinity of $R_{p}$, we have used a different approximation. This value was obtained from the complementary blistering study (see Sec. II for experimental details). The results of statistical analysis (not shown here) indicate that the amount of $\mathrm{H}_{2}$ in the blisters corresponds to about $60 \%$ of the molecular hydrogen obtained by SIMS technique for the bonded samples.

For the initial condition, i.e., the as-implanted state, the microcrack embryo is approximated by a platelet of $10 \mathrm{~nm}$ in diameter and $1 \mathrm{~nm}$ in thickness [see Fig. 7(a) and Ref. 5]. To simulate the growth of a larger (radius $>1 \mu \mathrm{m}$ ) microcrack from a platelet during the initial stage of annealing the following assumptions have been made: (1) there are no $\mathrm{H}_{2}$ molecules in the platelet in the as-implanted state; (2) the number of the $\mathrm{H}_{2}$ molecules in the microcrack after annealing for $t_{a}=10 \%$ of $t_{s}(T)$ was obtained using the experimental density of the microcracks [see Fig. 11(b)]; (3) based on the XTEM observation the defect thickness was assumed to "increase" from 1 to $2 \mathrm{~nm}$. Figure 12 shows that the calculated radius of a microcrack is in a good agreement with the experimental value for the average radius of the population for $10 \%$ of $t_{s}(T)$.

The same approach was used to fit the experimental data points for $t_{a}>10 \%$ of $t_{s}(T)$. The only fitting parameter was the microcrack thickness varied from 2 to $6 \mathrm{~nm}$ as a linear function of the annealing time. Figure 12 shows a good agreement between experiments and simulations for $t_{a}$ up to $80 \%$ of $t_{s}(T)$. For longer time there are small deviations between the model and the experiments. The range of the microcrack thickness used to fit the experimental data was the same for all temperatures studied in this paper.
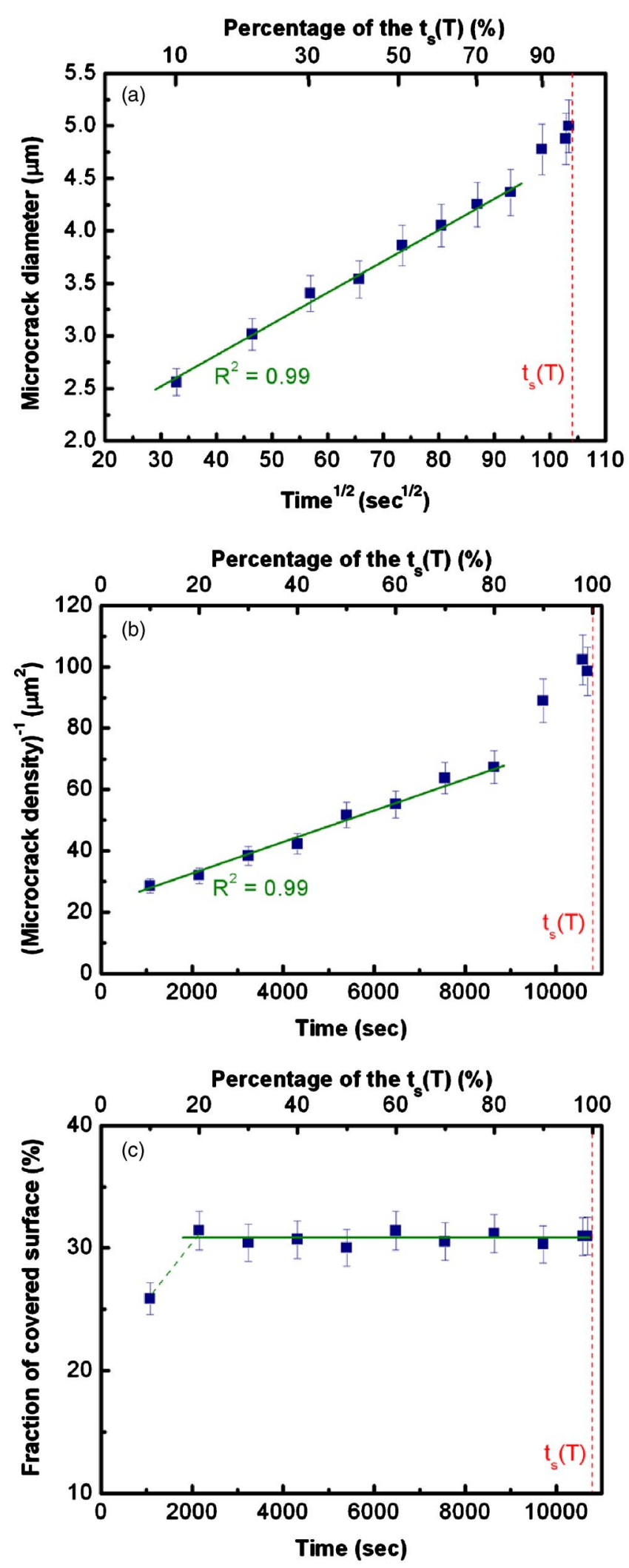

FIG. 11. (Color online) Dependence of the mean diameter of the microcrack population on the square root of the annealing time (a) at $400{ }^{\circ} \mathrm{C}$. Corresponding time dependences of the inverse surface density (b) and fraction of covered surface (c).

\section{DISCUSSION}

The presented results support the following scenario of the fracture phenomenon in $\mathrm{H}$ implanted (001) Si. In the 


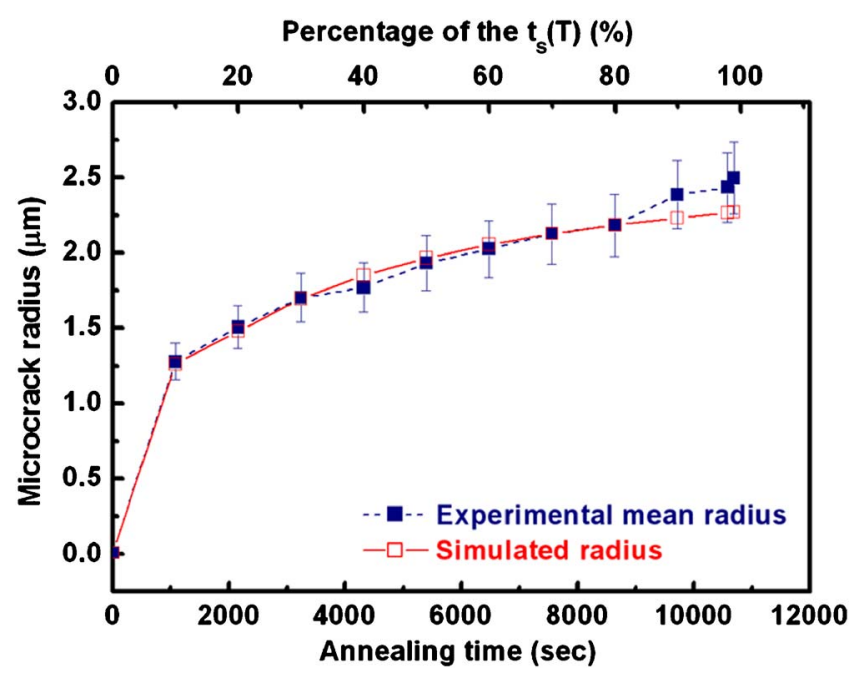

FIG. 12. (Color online) Time dependence of the microcrack radius for annealings at $400{ }^{\circ} \mathrm{C}$ : experimental data for the average size of the population and simulation results for the individual microcracks based on Griffith criterion.

as-implanted state, the damage zone is composed of numerous HPDs and platelets. The majority of platelets are on (001) plane parallel to the wafer surface. During the first period of the isothermal annealing a fast dissolution of the unstable HPDs takes place. The dominating precipitation phenomenon is characterized by the three simultaneous mechanisms.

(i) The growth of platelet by a crack propagation mechanism induced by the fast arrival of molecular $\mathrm{H}_{2}$.

(ii) The random nucleation of platelets, which is not entirely completed during the ion implantation.

(iii) Ostwald ripening for the platelet population in the damage zone.

After the end of the transient stage, i.e., $t_{a} \sim t_{s}(T) / 10$, buried microcracks, with the size $>1 \mu \mathrm{m}$, are detected. We believe that a microcrack forms as a result of the coalescence of numerous platelets. These "precursor" platelets have been nucleated and grown in close proximity and at about the same depth: around $R_{p}$. The platelets predominantly grow by crack propagation mechanism due to $\mathrm{H}_{2}$ injection at the beginning of annealing. They mechanically interact and this catastrophic interaction gives rise to the formation of an individual microcrack. This is not a common event as it can be judged from the respective densities of platelets and microcracks. There are about $1 \times 10^{6}$ less microcracks than platelets in the Si matrix during annealing. We can assume that the probability to form these large microcracks at the beginning of the annealing increases with the initial platelet density and thus with the implanted dose. These conditions are better met in the region close to $R_{p}$ where the density of platelets and $\mathrm{H}_{2}$ is higher and lead to the preferential formation of microcracks at that depth. For this reason, the microcracks have very narrow depth distribution as compared to those for platelets. As a consequence, two populations of HEDs coexist in the damage zone after initial stage of annealing: platelets with a mean diameter of $20 \mathrm{~nm}$ and microcracks with an average size of $2.5 \mu \mathrm{m}$.
XTEM analysis shows that for $t_{a}>t_{s}(T) / 10$ the platelets within the damage zone grow via conservative Ostwald ripening mechanism. The microcracks growth for $t_{a}$ between $10 \%$ and $80 \%$ of $t_{s}(T)$ is governed by a complex process involving different phenomena: an Ostwald ripening mechanism is coupled with a crack propagation process. The dissolution of the smaller microcracks leads to the continuous formation of $\mathrm{H}_{2}$ molecules within the bigger ones. The more developed microcracks grow by a crack propagation mechanism due to the high hydrogen gas pressure. In a good agreement with our model described in Sec. III D and our previous results ${ }^{6}$ the driving force behind the layer transfer is the formation of $\mathrm{H}_{2}$ in HEDs. The propagation mechanism of the individual microcracks leads to a random coalescence process between neighboring larger microcracks. We have shown in Sec. III C that this phenomenon occurs as the result of the sudden overlapping of their strain fields.

The strong impact of the coalescence on the statistical parameters of the microcrack population becomes evident at the end of the thermal treatment for $t_{a}>80 \%-90 \%$ of $t_{s}(T)$ when a growth mode changes. At this stage a strong direct interaction between the HEDs leads to an "accelerated" growth of the microcracks manifesting itself through the fast decrease of their density and the rapid increase of their mean size. The coalescence process due to the rupture of the $\mathrm{Si}$ lattice becomes predominant, leading to the full layer transfer. A model based on the Griffith criterion cannot describe the increase of the mean radius related to this mechanism since it does not take into account direct interactions between microcracks. A more sophisticated model describing the microcrack interaction during the catastrophic process occurring at the later stages of annealing (Fig. 12) has to be developed.

\section{SUMMARY}

The evolution of the $\mathrm{H}$ implant-induced defects in $\mathrm{Si}$ during isothermal annealings has been studied. The transient phenomenon during the initial stages of annealing is characterized by the fast dissolution of HPDs with about $60 \%$ of the implanted hydrogen involved. The predominant chemical reaction during this process corresponds to the formation of molecular hydrogen. The molecular $\mathrm{H}_{2}$ formation is not a linear function of the annealing time and has two regimes: a "fast-rate" domain due to dissociation of the hydrogenated complexes and a "slow-rate" domain occurring during remaining part of the thermal treatment.

Growth kinetics of HEDs can be divided into three stages. During the first $10 \%$ of the splitting time, the transient process of $\mathrm{H}_{2}$ formation induces the development of two distinct HED populations: platelet-type defects and microcracks of several microns in diameter. The fracture plane therefore is already defined during the first stage of annealing. For longer annealings, between $10 \%$ and $80 \%$ of the $t_{s}(T)$, the smaller HEDs, platelet grow via an Ostwald ripening mechanism. For larger HEDs, microcracks, the growth mechanism is more complex involving besides Ostwald ripening the crack propagation and coalescence. It is driven by the continuous $\mathrm{H}_{2}$ formation. In the last stage of annealing, 
the larger microcracks under high hydrogen gas pressure have strong mechanical interactions by the sudden overlapping of their strain fields leading to a predominant geometric coalescence process. This "catastrophic" mechanism results in the full layer transfer of $\mathrm{Si}$.

\section{ACKNOWLEDGMENTS}

F. Laugier is acknowledged for SIMS analysis and $\mathrm{N}$. Rochat for the help with the infrared spectroscopy measurements.

${ }^{1}$ M. Bruel, Electron. Lett. 31, 1201 (1995).

${ }^{2}$ M. K. Weldon, V. E. Marsico, Y. J. Chabal, A. Agarwal, D. J. Eaglesham, J. Sapjeta, W. L. Brown, D. C. Jacobson, Y. Caudano, S. B. Christman, and E. E. Chaban, J. Vac. Sci. Technol. B 15, 1065 (1997).

${ }^{3}$ N. M. Johnson, F. A. Ponce, R. A. Street, and R. J. Nemanich, Phys. Rev. B 35, 4166 (1987).

${ }^{4}$ J. Grisolia, G. Ben Assayag, A. Claverie, B. Aspar, C. Lagahe, and L. Laanab, Appl. Phys. Lett. 76, 852 (2000).

${ }^{5}$ B. Aspar, H. Moriceau, E. Jalaguier, C. Lagahe, A. Soubie, B. Biasse, A. M. Papon, A. Claverie, J. Grisolia, G. Benassayag, F. Leterte, O. Rayssac, T. Barge, C. Maleville, and B. Ghyselen, J. Electron. Mater. 30, 834 (2001).

${ }^{6}$ S. Personnic, A. Tauzin, K. K. Bourdelle, F. Letertre, N. Kernevez, F. Laugier, N. Cherkashin, A. Claverie, and R. Fortunier, AIP Conf. Proc.
866, 65 (2006)

${ }^{7}$ P. Holliger, F. Laugier, and J. C. Dupuy, Surf. Interface Anal. 34, 472 (2002).

${ }^{8}$ GRAMS software, http://www.thermo.com/com/CDA/.

${ }^{9}$ B. Bech Nielsen, L. Hoffmann, M. Budde, R. Jones, J. Goss, and S. Öberg, Mater. Sci. Forum 196-201, 933 (1995).

${ }^{10}$ M. Shinohara, T. Kuwano, Y. Akama, Y. Kimura, and M. Niwano, J. Vac. Sci. Technol. A 21, 25 (2003).

${ }^{11}$ M. Suezawa, Jpn. J. Appl. Phys., Part 2 37, L806 (1998).

${ }^{12}$ S. P. Timoshenko, Resistance Des Matériaux, Tome II (Dunod, Paris, 1981).

${ }^{13}$ C. M. Varma, Appl. Phys. Lett. 71, 3519 (1997).

${ }^{14}$ S. M. Myers, D. M. Follstaedt, H. J. Stein, and W. R. Wampler, Phys. Rev. B 47, 13380 (1993).

${ }^{15}$ P. Deak, L. C. Snyder, and J. W. Corbett, Phys. Rev. B 37, 6887 (1988).

${ }^{16}$ S. K. Estreicher, J. L. Hastings, and P. A. Fedders, Phys. Rev. B 57, R12 663 (1998).

${ }^{17}$ M. Kitajima, K. Ishioka, and S. Tateishi, Mater. Sci. Eng., B 58, 13 (1999).

${ }^{18}$ K. Ishioka, M. Kitajima, S. Tateishi, K. Nakanoya, N. Fukata, T. Mori, K. Murakami, and S. Hishita, Phys. Rev. B 60, 10852 (1999).

${ }^{19}$ T. Mori, K. Otsuka, N. Umehara, K. Ishioka, M. Kitajima, S. Hishita, and K. Murakami, Physica B 308-310, 171 (2001).

${ }^{20}$ W. Düngen, R. Job, Y. Ma, Y. L. Huang, T. Mueller, W. R. Fahrner, L. O. Keller, J. T. Horstmann, and H. Fieldler, J. Appl. Phys. 100, 034911 (2006).

${ }^{21}$ F. A. Reboredo, M. Ferconi, and S. T. Pantelides, Phys. Rev. Lett. 82, 4870 (1999).

${ }^{22}$ L. B. Freund, Appl. Phys. Lett. 70, 3519 (1997). 